\title{
Minimal residual disease monitoring in early stage follicular lymphoma can predict prognosis and drive treatment with rituximab after radiotherapy
}

\author{
Alessandro Pulsoni, ${ }^{1}$ (iD Irene Della \\ Starza, ${ }^{1}$ (D) Luca V. Cappelli, ${ }^{1}$ \\ Maria E. Tosti, ${ }^{2}$ Giorgia Annechini, ${ }^{1}$ \\ Marzia Cavalli, ${ }^{1}$ (D) Lucia A. De Novi, ${ }^{1}$ \\ Gianna M. D'Elia, ${ }^{1}$ Lavinia Grapulin, ${ }^{3}$ \\ Anna Guarini, ${ }^{4}$ Ilaria Del Giudice ${ }^{1}$ (iD \\ and Robin Foà ${ }^{1}$ \\ ${ }^{1}$ Haematology, Department of \\ Translational and Precision Medicine, \\ Sapienza University, ${ }^{2}$ National Centre for \\ Global Health, Istituto Superiore di Sanità, \\ ${ }^{3}$ Department of Radiology and \\ Radiotherapy, Sapienza University and \\ ${ }^{4}$ Department of Molecular Medicine, \\ Sapienza University, Rome, Italy \\ Received 10 January 2019; accepted for \\ publication 21 May 2019 \\ Correspondence: Alessandro Pulsoni, \\ Haematology, Department of Translational and \\ Precision Medicine, Sapienza University, Via \\ Benevento 6, 00161 Rome, Italy. \\ E-mail: alessandro.pulsoni@uniromal.it
}

\begin{abstract}
Summary
Since 2000, we have investigated 67 consecutive patients with stage I/II follicular lymphoma (FL) for the presence of BCL2/IGH rearrangements by polymerase chain reaction (PCR), real time quantitative PCR (RQ-PCR) and digital droplet PCR (ddPCR). All patients were treated with involvedfield radiotherapy (IF-RT) (24-30 Gy). From 2005, patients with minimal residual disease (MRD) after IF-RT received rituximab (R) $\left(375 \mathrm{mg} / \mathrm{m}^{2}, 4\right.$ weekly administrations). The median follow-up is 82 months (17-196). At diagnosis, $72 \%$ of patients were $B C L 2 / I G H+$. Progression-free survival (PFS) was significantly better in patients with undetectable/low levels $\left(<10^{-5}\right)$ of circulating $B C L 2 / I G H+$ cells at diagnosis and in those who were persistently MRD- during follow-up $(P=0.0038)$. IF-RT induced an MRD - status in 50\% of cases; $16 / 19$ (84\%) MRD+ patients after IF-RT became MRD - after R treatment. A significantly longer PFS was observed in $\mathrm{MRD}+$ patients treated with $\mathrm{R}$ compared to untreated $\mathrm{MRD}+$ patients $(P=0.049)$. In early stage FL, both circulating levels of $B C L 2 / I G H+$ cells at diagnosis and MRD status during follow-up bear prognostic implications. Standard IF-RT fails to induce an MRD-negative status in half of patients. Most patients become MRD- following treatment with $\mathrm{R}$ and this is associated with a significantly better PFS.
\end{abstract}

Keywords: early stage follicular lymphoma, radiotherapy, rituximab, MRD.
Follicular lymphoma (FL) is the second most common lymphoma in the United States and Western Europe, with a median age at diagnosis of 60 years (Anderson et al, 1998; Freedman, 2014; Al-Hamadani et al, 2015). About 20\% of FL patients are diagnosed in early stage (Armitage \& Weisenburger, 1998). Radiation therapy (RT) is the treatment of choice and results in 10-year overall survival (OS) rates of 60-80\%, with a median survival of approximately 19 years (Mac Manus \& Hoppe, 1996; Campbell et al, 2010; Pugh et al, 2010; Sancho et al, 2015). An initial observational strategy has also been considered (Friedberg et al, 2012; Barzenje et al, 2015). However, an analysis of the Surveillance, Epidemiology, and End Results Program registry and another recent study of the National Cancer Data Base on a very large number of patients have documented a significant advantage in OS in patients with early stage FL treated with RT compared to observation (Pugh et al, 2010; Vargo et al, 2015). Despite the impact of rituximab (R) treatment on the outcome of advanced stage FL, as well as on all other B-cell lymphomas (Hiddemann et al, 2005), due to the rarity of the disease only a few retrospective studies have suggested a role for R in early stage FL (Janikova et al, 2015; Ruella et al, 2016). As a result, the current European Society for Medical Oncology guidelines suggest involved field-RT (IF-RT) as the preferred upfront treatment option in patients with early stage $\mathrm{FL}$, reserving watchful waiting and $\mathrm{R}$ only to selected cases (Dreyling et al, 2016). These guidelines are however not universally followed, because many patients are not treated with IF-RT (Wennekes et al, 2011).

The genetic hallmark of FL is the translocation $\mathrm{t}(14 ; 18)$ (q32;q21) that causes the juxtaposition of the B-cell lymphoma/leukaemia 2 (BCL2) oncogene to the immunoglobulin heavy chain gene $(I G H)$ that results in an overexpression of the BCL2 protein and, consequently, in the clonal deregulation of cell cycle control and apoptosis (Klein \& Dalla-Favera, 2008). To monitor the disease, the BCL2/IGH 
rearrangement is widely exploited by polymerase chain reaction (PCR) amplification of the major breakpoint region (MBR), minor cluster region (mcr) (von Neuhoff et al, 1998) and $3^{\prime} \mathrm{MBR} / 5^{\prime} \mathrm{mcr}$ (minor BCL2 rearrangements) (Weinberg et al, 2007) in the peripheral blood (PB) and/or bone marrow $(\mathrm{BM})$, providing a sensitive molecular tool for minimal residual disease (MRD) evaluation in FL (Weinberg et al, 2007; Ladetto et al, 2013; Galimberti et al, 2014). The real time TaqMan PCR approach (RQ-PCR) is used for the quantification of the MBR rearrangements, while no validated assay is yet available for the study of the other breakpoints. A third generation quantitative PCR is represented by droplet digital PCR (ddPCR), which seems to be a reliable tool with a greater accuracy for the detection and quantification of molecular targets (Drandi et al, 2015).

In localised FL, despite a negative bone marrow biopsy, tumour cells can contaminate the $\mathrm{PB}$ and/or BM in about $60 \%$ of patients at diagnosis (Pulsoni et al, 2007). This is relevant in terms of lymphoma physiopathology and to better define the extent of disease dissemination at presentation (Mamessier et al, 2014). In addition, our group has shown that IF-RT of the primary site of the disease is capable of clearing BCL2/IGH+ cells from the $\mathrm{PB}$ and $\mathrm{BM}$ in more than $50 \%$ of cases, when the basal level of circulating lymphoma cells is $<1 \times 10^{-5}$ (Pulsoni et al, 2007).

In the present study, we analysed the prognostic impact of molecular tumour burden quantification at diagnosis and of MRD monitoring in a monocentric series of patients with early stage FL, and explored the possibility of an MRDguided therapeutic approach with $\mathrm{R}$. The aim was to better identify patients failing to respond optimally to IF-RT and who could benefit from further treatment.

Although the role of $\mathrm{R}$ is well established in advanced stage FL, no data are currently available in the setting of early stage FL.

\section{Methods}

This study is based on a retrospective series of consecutive patients diagnosed with stage I/II FL between 2000 and 2016 at a single institution. The present cohort represents the extension of our previously reported series (Pulsoni et al, 2007). Computed tomography (CT) or (in patients diagnosed since 2011) positron emission tomography (PET)/CT scan and BM biopsy were used for patients staging. Stage II patients were only selected if the involved areas were included in one RT field. All patients were treated with IF-RT using a $6 \mathrm{MeV}$ linear accelerator (24-30 Gy) (Lowry et al, 2011); from 2005, we introduced $\mathrm{R}$ consolidation $\left(375 \mathrm{mg} / \mathrm{m}^{2}, 4\right.$ weekly administrations) in patients who were persistently MRD+ after RT, in order to reduce or eliminate MRD (Fig 1). At diagnosis, $\mathrm{PB}$ and/or $\mathrm{BM}$ samples from all patients were analysed by nested PCR (N-PCR) to identify the presence of the MBR and mcr rearrangements (Gribben et al, 1991). Only in patients positive at baseline, the analysis was repeated after
IF-RT and, subsequently, every 6 months to monitor MRD (Fig 1). Available paraffin-embedded patients' lymph nodes (LN) were analysed by N-PCR and Sanger sequencing to confirm the identity of the molecular marker detected in the PB and/or BM, and to identify truly localised rearrangements in cases that resulted marker negative in the $\mathrm{PB} / \mathrm{BM}$. To identify the possible presence of rearrangements not detected by $\mathrm{N}-\mathrm{PCR}$, the marker negative samples at baseline were retrospectively analysed by the minor subclusters $\left(3^{\prime} \mathrm{MBR}\right.$ and $5^{\prime} \mathrm{mcr}$ ) by PCR (Weinberg et al, 2007). The baseline MBRpositive $(\mathrm{MBR}+)$ samples available were investigated retrospectively by RQ-PCR as previously described (Ladetto et al, 2001; van der Velden et al, 2007), and by ddPCR (Cavalli et al, 2017). Patients were routinely followed by clinical examination and echotomography every 3 months for the first 2 years after the end of IF-RT and, subsequently, every 6 months. CT or PET/CT scans were performed in case of suspected disease progression or every year. The event "progression" was defined as the detection by CT of a LN enlargement suggestive of disease progression. For the most recent patients diagnosed with PET/CT, we maintained CT scans as the reference criteria for progression definition. The project was approved by the Institutional Review Board. All patients signed a written informed consent for IF-RT and R treatment.

\section{Statistical analysis}

$P$-values for differences in categorical variables were calculated using the Chi Squared test or the Fisher's Exact test. Differences in tumour burden (expressed as a continuous variable) according to clinical and molecular parameters were tested by the Mann-Whitney $U$ test. The analysed endpoint for surveillance analysis was progression-free survival (PFS), because only 3 deaths have occurred so far. PFS was defined as the time from the date of diagnosis to disease progression, death from any cause or last follow-up and calculated by using the Kaplan-Meier method (Data S1); the Log-rank test was used to evaluate differences between factors. Regarding PFS analysis by $\mathrm{R}$ administration, the Mantel-Byar test (Delgado et al, 2014) was used; in particular, for each patient, the time starts at diagnosis and all patients begin in the "non-R arm"; those who eventually start $\mathrm{R}$ treatment enter the " $\mathrm{R}$ arm" and remain there until event or censoring. Also, to evaluate the effect of MRD positivity during sequential monitoring, Mantel-Byar analysis was used to account for the changes in MRD status during follow-up (Delgado et al, 2014). All data were analysed using the Stata Statistical Software, version 13.1 (StataCorp. LLC, College Station, TX, USA.

\section{Results}

\section{Overall clinical results}

The median age (range) at enrolment of the 67 patients was 57 years (37-84), $25(37 \cdot 3 \%)$ were male and $42(62 \cdot 7 \%)$ were 


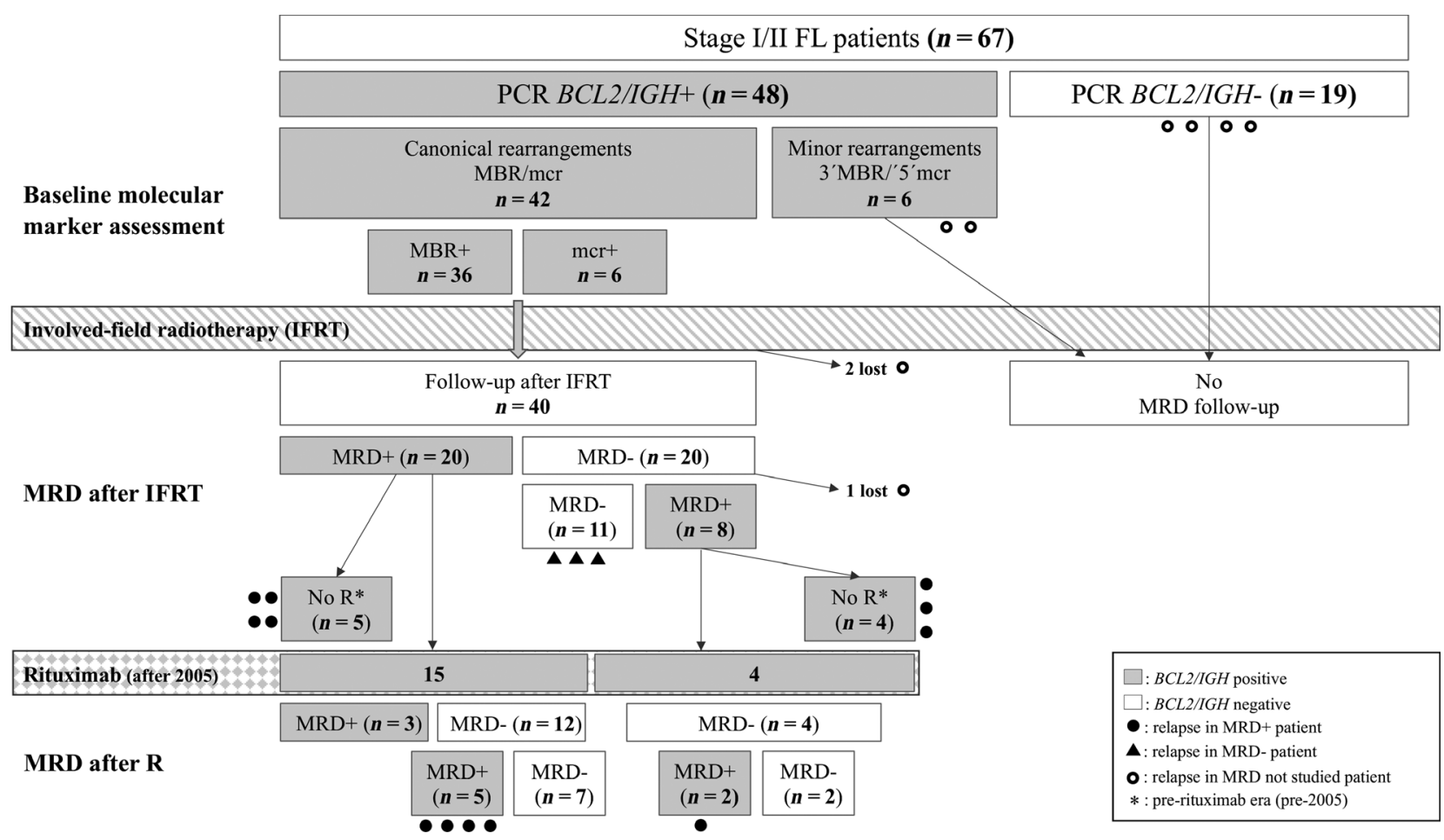

Fig 1. Flow chart of molecular evaluation, treatment plan and patient disposition. The figure shows the presence of BCL2/IGH markers among the 67 patients. Only patients with canonical $B C L 2 / I G H$ rearrangements $(n=42)$ were followed by MRD. All patients were treated with IF-RT and those who resulted MRD+ in the subsequent follow-up received $\mathrm{R}$ (after 2005). The figure also shows the distribution of relapses $(n=23)$ in the different subgroups of patients. FL, follicular lymphoma; IFRT, involved field radiotherapy; MBR, major breakpoint region; mcr, minor cluster region; MRD, minimal residual disease; PCR, polymerase chain reaction; R, rituximab.

female (Table I). According to the conventional staging procedures (Cheson et al, 2007), 52 patients were stage I $(77 \cdot 6 \%)$ and 15 stage II $(22 \cdot 4 \%)$. Overall, 11 patients were FL grade 1, 7 grade 1/2, 32 grade 2, 13 grade $3 \mathrm{~A}$ and 4 were not evaluable (Table I). The LN regions primarily involved by the disease were inguinal in $42 / 67(62.7 \%)$ patients, axillary in $7(10.4 \%)$, of the head-neck area (supraclavicular, later cervical, occipital, retro-auricular) in 15 (22.4\%), 1 epitrochlear and 2 extra-nodal (mammary) (4.5\%).

According to the FL International Prognostic Index (FLIPI; Solal-Céligny et al, 2004), 39 patients (58.2\%) had a score of $0,26(38.8 \%)$ of 1 and $2(3.0 \%)$ of 3.

Comparing the group of patients staged by CT alone versus PET/CT, no significant differences were observed according to the FLIPI score, nodal site, presence of $B C L 2 / I G H$ rearrangement at diagnosis by PCR or levels by RQ-PCR and ddPCR, and clinical outcome (data not shown).

Treatment consisted of IF-RT in all patients, followed by an MRD-driven $\mathrm{R}$ consolidation in 19 (28.4\%). All patients achieved a complete clinical response after IF-RT, defined as a reduction of the greatest LN transverse diameter to $\leq 1.5 \mathrm{~cm}$. Twenty-three $(34 \cdot 3 \%)$ of the 67 patients experienced a relapse, after a median of 37 months (range 9-130) (Fig 1).

As a result of the overall treatment strategy, PFS at 84 months was 63\% (95\% confidence interval: $49-75 \%)$ and
OS $100 \%$ (Figure S1). The median follow-up was 82 months (range 17-196). Three patients died of other causes: 1 developed a breast cancer which occurred in proximity to the radiation field (axilla) after a latency of 7 years; the other 2 developed a gastric cancer and a breast cancer which were outside of the RT field and were thus considered unrelated in both cases. PFS was not significantly different according to gender $(P=0 \cdot 74)$, stage I and II patients $(P=0 \cdot 25)$, histological grade $(P=0.43)$, nodal site $(P=0.73)$ or FLIPI score $(P=0 \cdot 71)$.

\section{Baseline molecular results and clinical correlation}

At baseline, a clonal marker was found in the $\mathrm{PB}$ and/or BM in $48 / 67$ cases $(71.6 \%)$ by PCR: 36 were MBR+ $(53.7 \%)$ and $6 \mathrm{mcr}+$ (9\%), while 6 showed a minor BCL2 rearrangement (9\%), retrospectively identified. The remaining 19 cases were negative (28.4\%). We always refer to the combination of $\mathrm{PB} / \mathrm{BM}$, because most cases proved concordant in the two compartments. Further details are provided in the Supplemental Material.

Paraffin-embedded LNs of 19/67 cases were analysed by PCR and Sanger sequencing, and 13 of these were evaluable. Eleven cases showed the same molecular marker identified in the $\mathrm{PB}$ and/or $\mathrm{BM}$, whilst 2 showed a BCL2 rearrangement (one MBR and one mcr) which was not found in the PB/BM. 
Table I. Patients' clinical features.

\begin{tabular}{|c|c|c|c|c|}
\hline & $\begin{array}{l}\text { Baseline } \\
\text { BCL2/IGH+ }\end{array}$ & $\begin{array}{l}\text { Baseline } \\
\text { BCL2/IGH- }\end{array}$ & $\begin{array}{l}P \text { - } \\
\text { value }\end{array}$ & Total \\
\hline $\begin{array}{l}\text { Number of } \\
\text { patients }\end{array}$ & $48(71 \cdot 6 \%)$ & $19(28.4 \%)$ & $0 \cdot 11$ & 67 \\
\hline $\begin{array}{l}\text { Age, years; } \\
\text { mean (range) }\end{array}$ & $58 \cdot 9(39-78)$ & $58 \cdot 6(37-84)$ & $0 \cdot 9$ & $58 \cdot 6(37-84)$ \\
\hline \multicolumn{5}{|l|}{ Sex } \\
\hline Male & $22(88 \%)$ & $3(12 \%)$ & 0.02 & $25(37 \cdot 3 \%)$ \\
\hline Female & $26(61.9 \%)$ & $16(38 \cdot 1 \%)$ & & $42(62 \cdot 7 \%)$ \\
\hline \multicolumn{5}{|l|}{ Ann Arbor stage } \\
\hline Stage I & $30(57 \cdot 7 \%)$ & $22(42 \cdot 3 \%)$ & $0 \cdot 14$ & $52(77 \cdot 6 \%)$ \\
\hline Stage II & $12(80 \%)$ & $3(20 \%)$ & & $15(22 \cdot 4 \%)$ \\
\hline \multicolumn{5}{|l|}{ FLIPI score } \\
\hline 0 & $28(71 \cdot 8 \%)$ & $11(28 \cdot 2 \%)$ & $0 \cdot 78$ & $39(58 \%)$ \\
\hline 1 & $19(73 \cdot 1 \%)$ & $7(26 \cdot 9 \%)$ & & $26(39 \%)$ \\
\hline 2 & $1(50 \%)$ & $1(50 \%)$ & & $2(3 \%)$ \\
\hline \multicolumn{5}{|l|}{ Grade } \\
\hline Grade 1 & $8(72 \cdot 7 \%)$ & $3(27 \cdot 3 \%)$ & $0 \cdot 6$ & $11(16 \cdot 4 \%)$ \\
\hline Grade $1 / 2$ & $6(85 \cdot 7 \%)$ & $1(14 \cdot 3 \%)$ & & $7(10 \cdot 4 \%)$ \\
\hline Grade 2 & $25(78 \cdot 1 \%)$ & $7(21 \cdot 9 \%)$ & & $32(47 \cdot 8 \%)$ \\
\hline Grade 3A & $8(61 \cdot 5 \%)$ & $5(38 \cdot 5 \%)$ & & $13(19 \cdot 4 \%)$ \\
\hline Not evaluable & $1(25 \%)$ & $3(75 \%)$ & & $4(6 \%)$ \\
\hline \multicolumn{5}{|l|}{ Primary location } \\
\hline Axillary & $6(85 \cdot 7 \%)$ & $1(14 \cdot 3 \%)$ & $0 \cdot 62$ & $7(10 \cdot 3 \%)$ \\
\hline Head-neck & $12(80 \%)$ & $3(20 \%)$ & & $15(22 \cdot 4 \%)$ \\
\hline Inguinal & $28(66 \cdot 7 \%)$ & $14(33 \cdot 3 \%)$ & & $42(62 \cdot 6 \%)$ \\
\hline Other & $2(71 \cdot 6 \%)$ & $1(28 \cdot 4 \%)$ & & $3(4 \cdot 7 \%)$ \\
\hline
\end{tabular}

FLIPI, follicular lymphoma international prognostic index.

Males showed a significantly higher probability of carrying the $B C L 2 / I G H$ rearrangement at baseline $(P=0 \cdot 02)$. Stage I FLs showed a marker in $36 / 52$ cases $(69 \%)$ and stage II in $12 / 15$ cases $(80 \%)(P=0.53)$ (Table I). Also, there was no statistical difference in the presence of the $B C L 2 / I G H$ rearrangement according to grade, nodal site and FLIPI score (Table I).

PFS was not significantly longer in patients negative at baseline for BCL2/IGH rearranged cells in the PB/BM: the PFS at 84 months was $75 \%(45-90 \%)$ in negative patients versus $59 \%(42-73 \%)$ in $B C L 2 / I G H$ positive patients $(P=0 \cdot 26)$.

\section{Tumour burden quantification at diagnosis: $R Q-P C R$} versus $d d P C R$

In addition to the baseline qualitative evaluation, we also retrospectively assessed the $B C L 2 / I G H$ levels at diagnosis in BM and $\mathrm{PB}$ samples of the MBR+ patients with available material using RQ-PCR and the new quantitative approach, ddPCR. The comparative analysis between ddPCR and RQ-PCR has already been reported by our group, indicating that ddPCR was comparable and potentially more accurate than RQ-PCR (Cavalli et al, 2017). RQ-PCR was performed on 30/36 MBR+ patients, 17 of which $(56 \cdot 7 \%)$ showed a tumour burden $\geq 10^{-5}$ (quantifiable), $8(26 \cdot 7 \%)$ were positive not quantifiable (PNQ, $\left.10^{-6}\right)$ and $5(16 \cdot 6 \%)$ were negative $\left(<10^{-5}\right)$. ddPCR was performed on the same cohort of patients with available samples: $19(63 \cdot 4 \%)$ showed a tumour burden $\geq 10^{-5}$ (quantifiable), 10 $(33 \cdot 3 \%)$ were PNQ $\left(10^{-6}\right)$ and $1(3 \cdot 3 \%)$ was negative $\left(<10^{-5}\right)$. Further details on the $\mathrm{PB} / \mathrm{BM}$ concordance in the RQ/ddPCR analysis are provided in the Data $\mathrm{S} 1$.

We then stratified patients according to disease level, i.e. as quantifiable $\left(\geq 10^{-5}\right)$ or negative/PNQ $\left(<10^{-5}\right)$ : the former group was associated (both by RQ-PCR and ddPCR) with a significantly weaker molecular response to RT $(P<0.003$ and $P=0 \cdot 048$, respectively) and MRD persistence during follow-up $(P=0.001$ and $P<0.001$, respectively) (Table S1). Moreover, a quantifiable disease burden at diagnosis was associated with a significantly worse PFS if investigated by ddPCR (84-month PFS $90.9 \%$ vs. $38.0 \%$ months, $P=0.015$ ) but not by RQ-PCR (84-month PFS $69.9 \%$ vs. $46 \cdot 5 \%$ months, $P=0$-087) (Fig 2A, B).

Finally, considering the baseline BCL2/IGH levels by ddPCR as a continuous variable, these were significantly associated with the MRD clearance after both RT $(P=0.010)$ (Fig 2C-2) and $\mathrm{R}(P=0.015)$ (Fig 2C-3), the MRD status in the follow-up $(P<0.001)$ (Fig 2C-4) and the relapse probability $(P=0.05)$ (Fig $2 \mathrm{C}-5)$. No association with the involved nodal site was found $(P=0.08)$ (Fig 2C$1)$, nor with stage or FLIPI score (data not shown).

\section{Clinical and molecular results after radiotherapy}

The irradiation of the involved LNs was followed by a complete clinical response (CR) in all patients. Of the 42 patients with canonical MBR and mcr BCL2/IGH rearrangements at baseline, 40 were molecularly evaluated for MRD after RT and in the subsequent follow-up (2 were lost because they refused further molecular analysis). Figure 1 shows the number of patients studied at each MRD time point, the MRD results and the distribution of clinical relapses.

IF-RT led to a disappearance of a detectable signal from the $\mathrm{PB} / \mathrm{BM}$ in half of previously positive patients: $20 / 40$ (50\%) of patients proved MRD-, while 20 were persistently MRD+. Regardless of the post-RT MRD status, an equal number of clinical relapses were recorded in both groups ( 8 each) and the time-to-event PFS analysis of post-RT MRD- versus MRD+ patients was not significantly different $(P=0 \cdot 7)$. In the subsequent follow-up, $1 \mathrm{MRD}$ - patient was lost (refused further molecular analysis) and an additional group of 8 patients who were MRD - immediately after RT became MRD+ after a median of $36 \cdot 2$ months (range 11.6-47.4) (Fig 1).

\section{Effect of rituximab treatment on MRD status and on PFS}

In an attempt to reduce the probability of relapse, $\mathrm{R}$ was administered to MRD+ patients after IF-RT. No statistical difference in gender, stage, FLIPI, grade and nodal site was observed between patients who received $\mathrm{R}$ and those who 
(A)

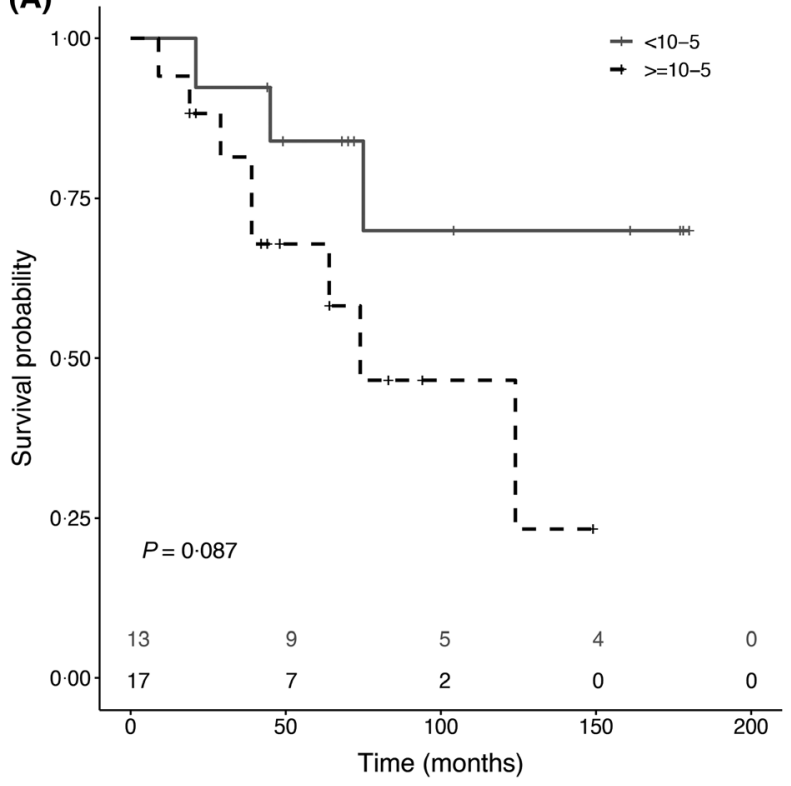

(B)

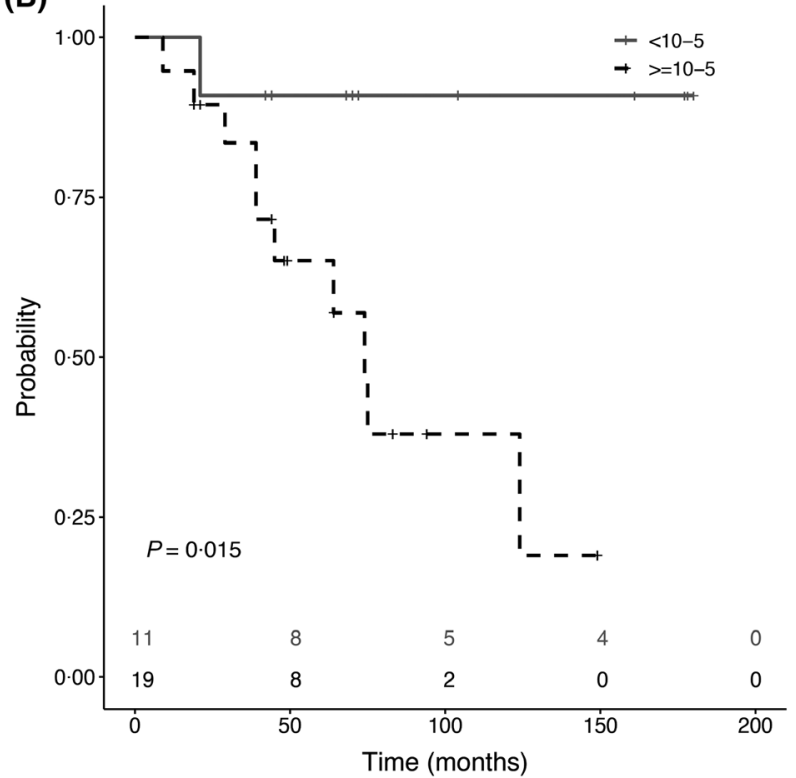

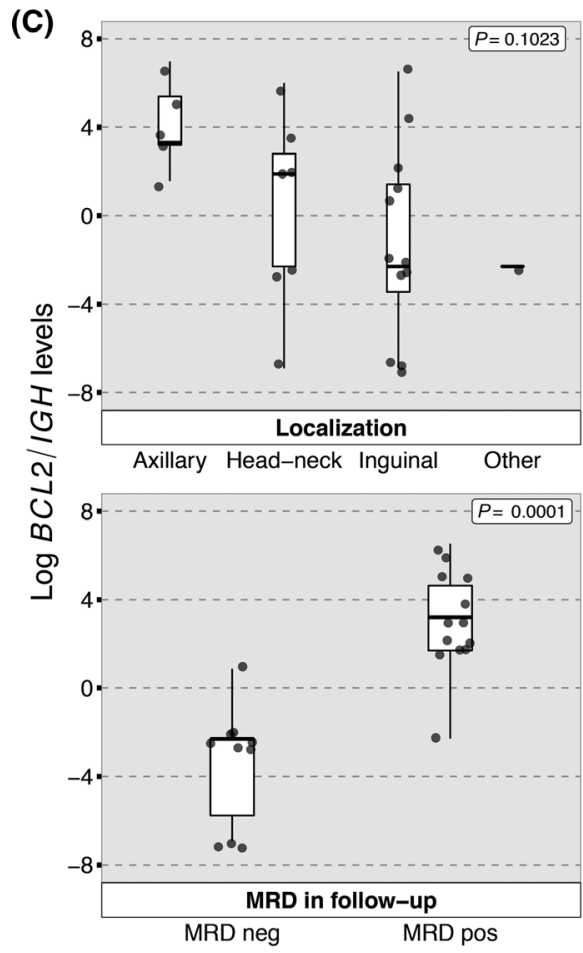
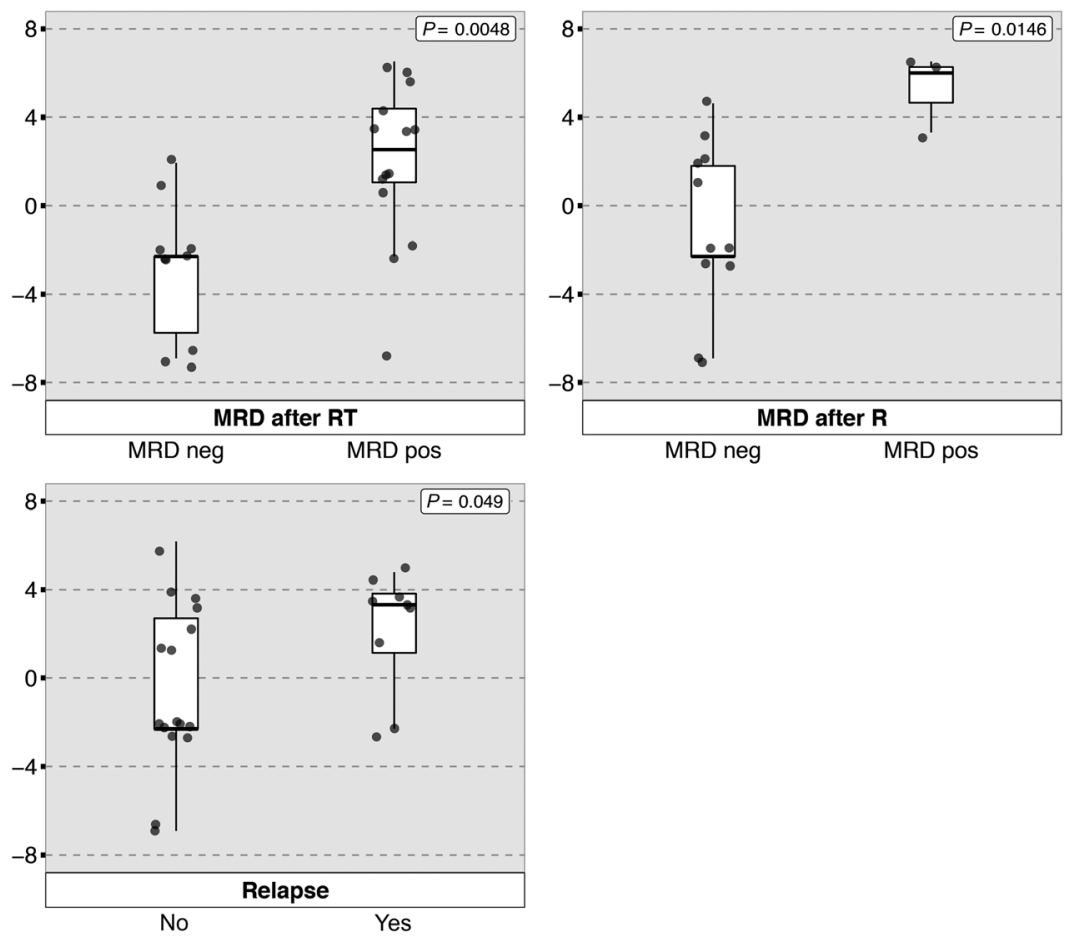

Fig 2. Clinical outcome according to the tumour burden at diagnosis quantified by (A) real time quantitative polymerase chain reaction and (B) digital droplet polymerase chain reaction ddPCR. (C) BCL2/IGH levels by ddPCR plotted with clinical parameters and MRD). (C1) Levels among different disease localization. (C2, C3, C4) Levels and MRD after RT, after R and during follow-up, respectively. (C5) Levels and relapse. MRD, minimal residual disease; R, rituximab; RT, radiotherapy.

did not (Table S2). Despite the earlier observation of the pre-2005 untreated cohort, the observation period was superimposable between the two groups (68 months in the $\mathrm{R}$ group vs. 60 in the untreated one, $P=0.862$ ). This was due to the higher proportion of relapsing cases in the untreated patients, with a consequent stop of their observation time.
Nineteen MRD+ patients received R: 15/19 were persistently MRD+ after IF-RT, whilst 4/19 received $\mathrm{R}$ after converting from MRD - to MRD+ during follow-up (Fig 1). In the first group, 12/15 patients converted to MRD- after R, while $3 / 15$ remained MRD+; in the second group, all patients (4/4) became MRD-. Overall, $\mathrm{R}$ induced a status of MRD 
negativity in $16 / 19$ treated patients (84\%). Seven of the 16 MRD - patients (43.7\%) became MRD+ after a median of 12 months (range 7-18) from $\mathrm{R}$ treatment and 5/7 (71\%) relapsed, whilst 9/16 remained persistently MRD- (56.3\%) and none has so far relapsed after a mean follow-up of 74 months (range 19-124) $(P=0 \cdot 005)$.

To evaluate the impact of $\mathrm{R}$ on clinical outcome, we considered the series of the $19 \mathrm{MRD}+$ patients who received $\mathrm{R}$ compared to the $9 \mathrm{MRD}+$ who did not (pre-2005) (Fig 1). Of the former 19 , only 5 have relapsed (26\%), whilst of the latter 9, 7 have relapsed $(78 \%)(P=0 \cdot 017)$. Considering that $\mathrm{R}$ was administered to patients at different times (when they were found MRD+ during follow-up), we performed a time-to-event analysis (Mantel-Byar test) using $\mathrm{R}$ as a time-dependent covariate. The difference in PFS between R-treated and R-untreated patients was statistically significant $(P=0.049)$ (Fig 3A). No grade 3-4 adverse events were recorded after the 4 weekly doses of R. No patient required treatment delay or interruption.

\section{MRD monitoring during follow-up predicts relapse}

To define the predictive role of MRD in the entire cohort regardless of treatment, we considered the group of $39 / 42$ $\mathrm{MBR} / \mathrm{mcr}+$ patients with available molecular follow-up (Fig 1). Overall, 19/39 remained or became MRD+ during followup and 20/39 were MRD-. Twelve of the $19 \mathrm{MRD}+$ patients $(63 \%)$ have relapsed, while this has occurred only in $3 / 20$ MRD - patients (15\%) $(P=0.002) \quad$ (Fig 1). As patients became MRD+ at different timepoints, we performed a Mantel-Byar estimate of PFS to take this diversity into account: PFS was significantly better for patients who remained persistently $\mathrm{MRD}-$ compared to $\mathrm{MRD}+$ patients (median not reached vs. 67 months $)(P=0 \cdot 0038)$ (Fig 3B).

\section{Discussion}

Very limited data are available on the relevance of $B C L 2 / I G H$ molecular quantification at diagnosis and of MRD monitoring following treatment in early stage FL. In this study, which expands our previous observations (Pulsoni et al, 2007), we were able to identify three different scenarios in which a localised FL can be found according to the molecular tumour burden at diagnosis (Fig 4).

\section{Early stage FL with undetectable circulating BCL2/IGH+ cells}

We had clinical evidence of this disease confined to the LN in 2 of the 13 cases in which a molecular study of the biopsied LN was possible: they were positive in the LN for the BCL2/IGH translocation, while negative in the PB/BM. Both patients, diagnosed in 2014, underwent IF-RT that probably eradicated the disease since they remained MRD- and have not yet relapsed.

\section{Early stage FL with low circulating tumour burden}

In these cases, only a small proportion of FL cells could be found outside the $\mathrm{LN}\left(<1 \times 10^{-5} \mathrm{BCL} 2 / \mathrm{IGH}+\right.$ cells $)$. This very low circulating tumour burden may still be eradicated, because it was associated with a better molecular response to IF-RT treatment. Moreover, these patients, together with
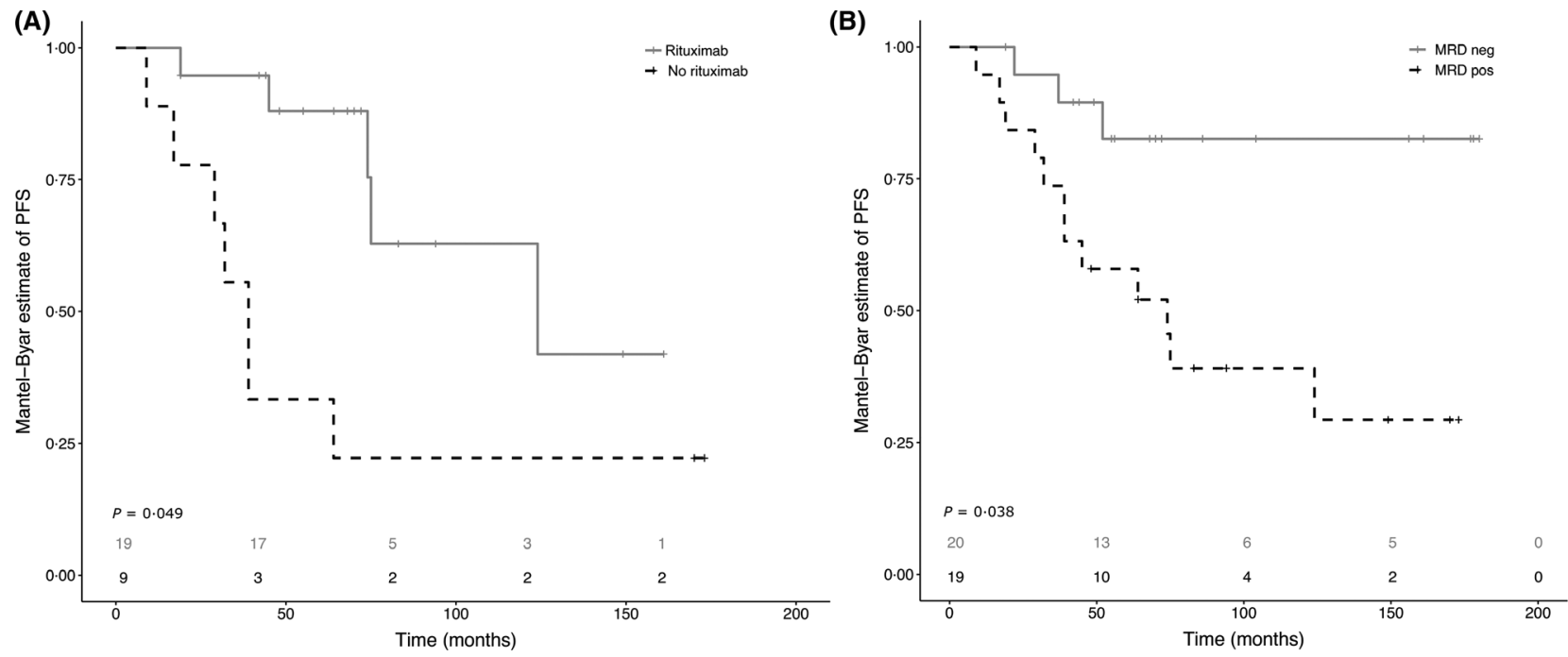

Fig 3. Role of R consolidation in MRD+ patients (A) and MRD assessment in the follow-up (B). (A) (Mantel-Byar test) shows the impact of rituximab (R) in the context of those patients who remained minimal residual disease-positive (MRD+) after involved field radiotherapy (IF-RT). Patients treated with $\mathrm{R}$ were compared with those, observed before 2005, who did not receive it. (B) (Mantel-Byar test) shows the progression-free survival (PFS) of minimal residual disease-negative (MRD-) patients versus MRD+ patients during follow-up. The MRD- group includes patients who became negative after IF-RT, as well as those who became MRD - after R treatment and maintained it in multiple follow-up determinations. 


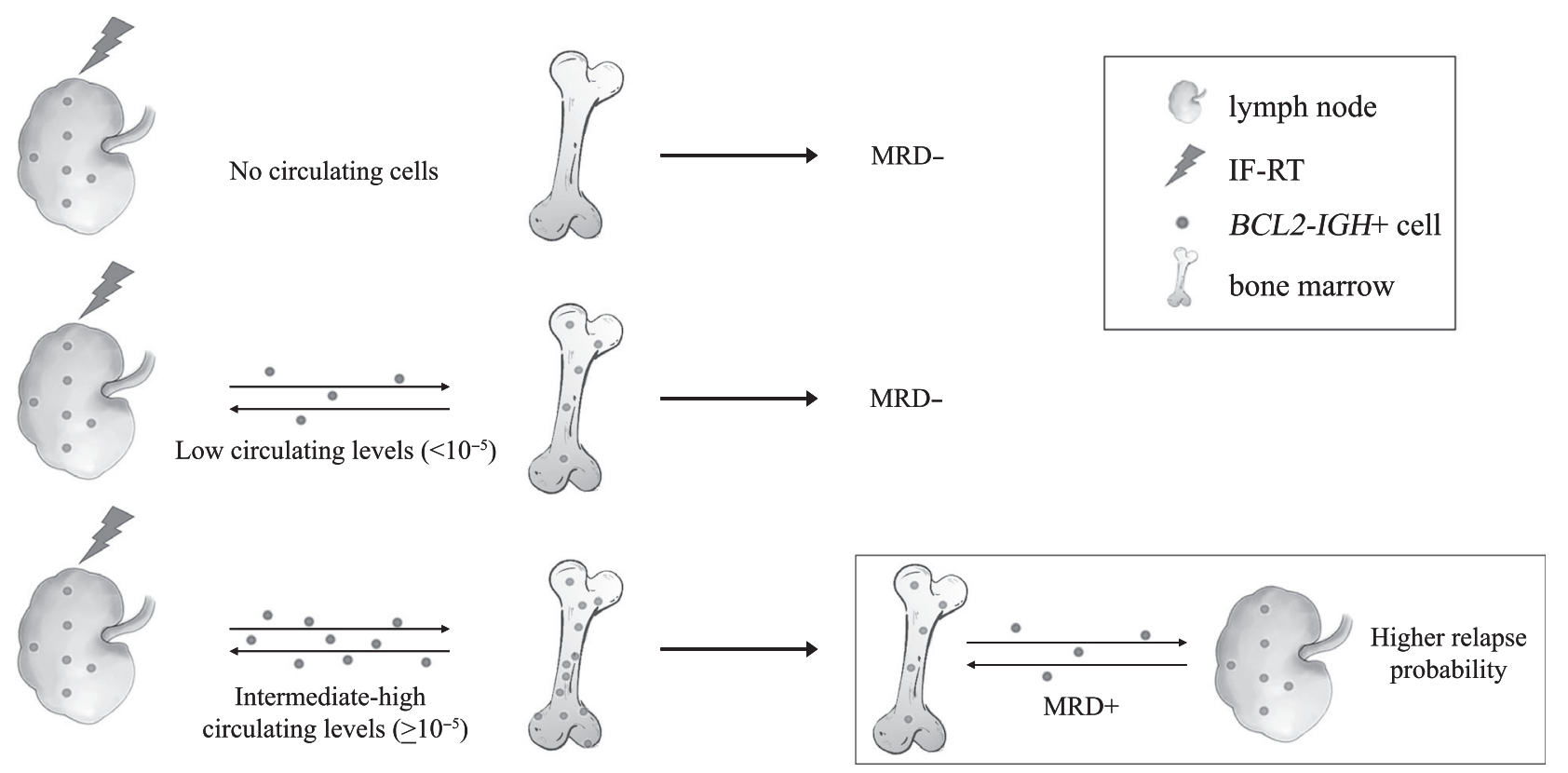

Fig 4. Three different scenarios in which an early stage follicular lymphoma can be found. If the BCL2/IGH circulating levels are absent/low $\left(<10^{-5}\right)(\mathrm{A}, \mathrm{B})$, the chances of obtaining a minimal residual disease-negative (MRD-) status after involved field radiotherapy (IF-RT) are significantly higher and the progression-free survival is better. Quantifiable disease levels $\left(\geq 10^{-5}\right)$ (C) are associated with a worse prognosis and increased relapse probability.

those of the previous group, showed a significantly better PFS compared to the subsequent group.

\section{Early stage FL with intermediate-high circulating tumour burden}

In most cases, FL cells had already invaded the blood stream and the tumour burden levels were higher than in the previous groups $\left(\geq 1 \times 10^{-5} B C L 2 / I G H+\right.$ cells $)$. This third scenario may be considered as a sort of "borderline" localised FL, because, despite a negative BM biopsy, cases where FL cells have contaminated the $\mathrm{PB}$ and/or $\mathrm{BM}$ at the molecular level are likely to disseminate to distant LNs and/or extranodal sites, giving rise to an advanced FL.

Furthermore, a fourth group of patients is represented by those who did not constitutively express any detectable BCL2/IGH marker, whose tumour burden levels could not be evaluated with any of the available molecular techniques (19/ 67: $28 \%)$.

Thus, early stage FL can be considered the earliest phase of the multistep process of FL pathogenesis (Roulland et al, 2006; Klein \& Dalla-Favera, 2008; Kluin, 2013; Mamessier et al, 2014).

In advanced stage FL, increasing evidence suggests that the level of BCL2/IGH rearrangement in the $\mathrm{PB}$ and/or $\mathrm{BM}$ before treatment is of prognostic relevance (Rambaldi et al, 2005; Hirt et al, 2008; Galimberti et al, 2014). In the present study, we showed that in localised FL the BCL2/IGH pretreatment levels predicted patients' outcome, whilst other clinical markers did not. The PFS of patients with non-quantifiable disease at diagnosis $\left(<10^{-5}\right)$ by ddPCR was significantly better than that of patients with quantifiable levels $\left(\geq 10^{-5}\right)$, whilst by RQ-PCR only a trend was observed. Moreover, lower baseline BCL2/IGH levels were significantly associated with MRD clearance after both RT and R, and had a higher likelihood of remaining MRD- during the clinical follow-up. Accumulating evidence indicates that ddPCR is associated with less false positive results compared to RQPCR (Della Starza et al, 2018). We therefore suggest the possibility of using this new accurate approach as a prognostic tool to better predict the clinical outcome and more precisely define the molecular response of patients with early stage FL. For a direct comparison between ddPCR and RQ-PCR in this series of patients, see Cavalli et al (2017).

We also demonstrated that MRD assessment during the clinical follow-up is a powerful prognostic tool to define the outcome of patients affected by localised FL, regardless of the treatment received: MRD+ patients had indeed a significantly lower PFS compared to those who remained MRD-.

With regard to the treatment strategy for early stage FL, the validity of the standard approach by IF-RT has been questioned by some authors who reported similar survival results independently of the treatment strategy employed in early stage FL (Friedberg et al, 2012). An alternative approach with $\mathrm{R}$ alone could be thus considered in selected cases to spare radiotherapy. However, the potential role of IF-RT as a curative solution in localised FL has to be kept in mind, whilst no data are available in the literature on a definitive curative role of $\mathrm{R}$. Indeed, retrospective registry studies analysing a very large number of cases documented that upfront RT improves disease-specific survival (DSS) 
(Pugh et al, 2010) and OS (Pugh et al, 2010; Vargo et al, 2015) compared to watchful waiting, and that an inadequate initial treatment may be associated with a more unfavourable outcome. Very recent data have also shown that the addition of systemic therapy with 6 cycles of R-CVP (R plus cyclophosphamide, vincristine and prednisone) after IF-RT reduced relapses outside the radiation fields and significantly improved PFS compared to IR-FT alone in early stage FL (MacManus et al, 2018). Of note, also in this prospective trial the 10-year OS was not different when adding systemic therapy to IF-RT. Other groups have suggested the addition of R monotherapy to RT in early stage FL on the bases of retrospective series (Janikova et al, 2015; Ruella et al, 2016). None are based on a molecular tailored approach. In the present study, which reinforces and expands our earlier observations (Pulsoni et al, 2007), we showed that IF-RT was capable of inducing MRD negativity in 50\% of baseline BCL2/IGH-positive cases. This indicates that, although some FL cells may circulate outside the limits of the LNs even in patients with localised disease, early RT treatment may help to clear them by inducing a systemic immune response (known as abscopal effect) (Reynders et al, 2015). However, the molecular response to IF-RT was significantly worse in patients with quantifiable levels of $B C L 2 / I G H$ rearrangement at diagnosis, reinforcing the notion that RT is not always sufficient to prevent spreading of the primary disease (Friedberg et al, 2012; MacManus et al, 2018).

We support the idea of treating patients affected by early stage FL with anti-CD20 monoclonal antibodies added to IFRT (Witzens-Harig et al, 2011), aiming to defer, and potentially avoid, systemic chemotherapy. Our goal is to use MRD evaluation to better identify the proportion of patients not destined to be cured by RT. The addition of R to this subgroup potentially resistant to RT could theoretically increase the proportion of cured patients.

Despite the limits of this study, mainly residing in the heterogeneity and complexity of our series, with different subsets of patients, our data clearly show that the administration of $\mathrm{R}$ is capable of significantly reducing the likelihood of relapse in MRD+ patients and that the PFS of R-treated patients is significantly better than that of untreated patients. Moreover, if a patient achieves an MRD-negative status after having received $\mathrm{R}$ the probability of relapsing is significantly lower than that of a patient who remains MRD+ during follow-up. Finally, we could also demonstrate that the kinetics of MRD during the follow-up is a powerful prognostic tool to define the outcome of patients affected by localised FL, regardless of the treatment received: patients who remained or became MRD+ had a significantly lower PFS compared to those who remained MRD-. The integration of PET/CT scan with MRD monitoring could further improve the response assessment and outcome prediction, as suggested for advanced FL (Luminari, et al, 2016).

Although a benefit of adding $\mathrm{R}$ could have been predicted, given its well established role in the context of advanced stage
FL, to our best knowledge this is the first demonstration of its potential efficacy also in early stage FL. Although an improvement in PFS of R-treated patients does not necessarily imply a benefit in OS or in subsequent PFS2, our data, together with other preliminary experiences from other groups (WitzensHarig et al, 2011; Ruella et al, 2016), support the introduction of $\mathrm{R}$ consolidation also in early-stage FL.

Moreover, the results of the MRD-driven strategy suggest that persistent MRD negativity could possibly represent an endpoint of treatment for early stage FL patients. This approach could represent a cost and time saving strategy, because $\mathrm{R}$ would be administered only to patients who remain or become MRD+ during follow-up. The results of this study, together with our earlier preliminary experience (Pulsoni et al, 2007), led to the design of a prospective phase II Fondazione Italiana Linfomi trial (MIRO, EudraCT: 2012001676-11), aimed at conclusively demonstrating the benefit of the anti-CD20 monoclonal antibody ofatumumab in treating MRD+ patients after RT.

In conclusion, the results of this study indicate that: (i) localised FL is a rare and heterogeneous entity, associated with different levels of disease spread from the original LN at the time of diagnosis; (ii) the use of quantitative PCR for the detection of $B C L 2 / I G H$ circulating levels at diagnosis is a powerful tool to quantify the disease burden; this is associated both with the degree of molecular response to treatment (IF-RT and R) and with the probability of relapsing; (iii) ddPCR shows a higher level of accuracy compared to conventional RQ-PCR; iv) IF-RT alone is not always capable of clearing circulating FL cells; (v) a MRD-driven consolidation with $\mathrm{R}$ after standard IF-RT improves the PFS of patients with early stage FL.

\section{Acknowledgements}

The authors wish to thank Associazione Italiana per la Ricerca sul Cancro (AIRC) 5 x 1000, Milan, Italy (Special Program Molecular Clinical Oncology MCO-10007; Metastases Special Program, $N^{\circ}$ 21198) (Robin Foà); Progetti di Rilevante Interesse Nazionale (PRIN) 2015ZMRFEA (RF).

\section{Authorship}

Contribution: R.F. and A.P. designed the research; A.P., G.A., G.M.D'E. and L.G. were responsible for the clinical management of the patients; I.D.S., L.V.C., M.C., L.A.D.N. A.G., organized and performed the experimental laboratory procedures; M.E.T. and L.V.C. were responsible for the statistical analysis; A.P., I.D.S., L.V.C., I.D.G. and R.F. wrote the manuscript; R.F. critically revised the manuscript; all authors approved the final version of the manuscript.

\section{Conflict of interest}

The authors have no conflicts of interest to disclose. 


\section{Supporting Information}

Additional supporting information may be found online in the Supporting Information section at the end of the article.

Data S1. Concordance between peripheral blood (PB) and bone marrow (BM) by PCR and RQ-/ddPCR.
Table S1. BCL2/IGH levels by RQ-PCR/ddPCR and MRD clearance after IF-RT and in the follow-up.

Table S2. Patients' clinical features according to $\mathrm{R}$ treatment.

Fig S1. OS and PFS of the entire population.

\section{References}

Al-Hamadani, M., Habermann, T.M., Cerhan, J.R., Macon, W.R., Maurer, M.J. \& Go, R.S. (2015) Non-Hodgkin lymphoma subtype distribution, geodemographic patterns, and survival in the US: a longitudinal analysis of the National Cancer Data Base from 1998 to 2011. American Journal of Hematology, 90, 790-795.

Anderson, J.R., Armitage, J.O. \& Weisenburger, D.D. (1998) Epidemiology of the non-Hodgkin's lymphomas: distributions of the major subtypes differ by geographic locations. Non-Hodgkin's Lymphoma Classification Project. Annals of Oncology: Official Journal of the European Society for Medical Oncology, 9, 717-720.

Armitage, J.O. \& Weisenburger, D.D. (1998) New approach to classifying non-Hodgkin's lymphomas: clinical features of the major histologic subtypes. Non-Hodgkin's Lymphoma Classification Project. Journal of Clinical Oncology, 16, 2780-2795.

Barzenje, D.A., Cvancarova Smastuen, M., Liestol, K., Fossa, A., Delabie, J., Kolstad, A. \& Holte, H. (2015) radiotherapy compared to other strategies in the treatment of stage I/II follicular lymphoma: a study of 404 patients with a median follow-up of 15 years. PLoS One, 10, e0131158.

Campbell, B.A., Voss, N., Woods, R., Gascoyne, R.D., Morris, J., Pickles, T., Connors, J.M. \& Savage, K.J. (2010) Long-term outcomes for patients with limited stage follicular lymphoma: involved regional radiotherapy versus involved node radiotherapy. Cancer, 116, 3797-3806.

Cavalli, M., De Novi, L.A., Della Starza, I., Cappelli, L.V., Nunes, V., Pulsoni, A., Del Giudice, I., Guarini, A. \& Foa, R. (2017) Comparative analysis between RQ-PCR and digital droplet PCR of BCL2/IGH gene rearrangement in the peripheral blood and bone marrow of early stage follicular lymphoma. British Journal of Haematology, 177, 588-596.

Cheson, B.D., Pfistner, B., Juweid, M.E., Gascoyne, R.D., Specht, L., Horning, S.J., Coiffier, B., Fisher, R.I., Hagenbeek, A., Zucca, E., Rosen, S.T., Stroobants, S., Lister, T.A., Hoppe, R.T., Dreyling, M., Tobinai, K., Vose, J.M., Connors, J.M., Federico, M., Diehl, V.; for the International Harmonization Project on Lymphoma (2007) Revised response criteria for malignant lymphoma. Journal of Clinical Oncology, 25, 579-586.

Delgado, J., Pereira, A., Villamor, N., López-Guillermo, A. \& Rozman, C. (2014) Survival analysis in hematologic malignancies: recommendations for clinicians. Haematologica, 99, 1410-1420.

Della Starza, I., Del Giudice, I., Menale, L., Cappelli, L.V., Cavalli, M., De Novi, L.A., Apicella, V., Soscia, R., Cafforio, L., Mariglia, P., Chiaretti, S., Vitale, A., Guarini, A. \& Foà, R. (2018) Minimal residual disease (MRD) detection by digital-droplet-pcr (ddPCR) in lymphoid malignancies. Haematologica, 103, S1-S129.

Drandi, D., Kubiczkova-Besse, L., Ferrero, S., Dani, N., Passera, R., Mantoan, B., Gambella, M., Monitillo, L., Saraci, E., Ghione, P., Genuardi, E., Barbero, D., Omede, P., Barberio, D., Hajek, R., Vitolo, U., Palumbo, A., Cortelazzo, S., Boccadoro, M., Inghirami, G. \& Ladetto, M. (2015) Minimal residual disease detection by droplet digital PCR in multiple myeloma, mantle cell lymphoma, and follicular lymphoma: a comparison with real-time PCR. The Journal of Molecular Diagnostics, 17, 652-660.

Dreyling, M., Ghielmini, M., Rule, S., Salles, G., Vitolo, U., Ladetto, M. \& Committee, E.G. (2016) Newly diagnosed and relapsed follicular lymphoma: ESMO Clinical Practice Guidelines for diagnosis, treatment and follow-up. Annals of Oncology, 27, v83-v90.

Freedman, A. (2014) Follicular lymphoma: 2014 update on diagnosis and management. American Journal of Hematology, 89, 429-436.

Friedberg, J.W., Byrtek, M., Link, B.K., Flowers, C., Taylor, M., Hainsworth, J., Cerhan, J.R., Zelenetz, A.D., Hirata, J. \& Miller, T.P. (2012) Effectiveness of first-line management strategies for stage I follicular lymphoma: analysis of the National LymphoCare Study. Journal of Clinical Oncology, 30, 3368-3375.

Galimberti, S., Luminari, S., Ciabatti, E., Grassi, S., Guerrini, F., Dondi, A., Marcheselli, L., Ladetto, M., Piccaluga, P.P., Gazzola, A., Mannu, C., Monitillo, L., Mantoan, B., Del Giudice, I., Della Starza, I., Cavalli, M., Arcaini, L., Tucci, A., Palumbo, G.A., Rigacci, L., Pulsoni, A., Vitolo, U., Boccomini, C., Vallisa, D., Bertoldero, G., Gaidano, G., Musto, P., Petrini, M. \& Federico, M. (2014) Minimal residual disease after conventional treatment significantly impacts on progression-free survival of patients with follicular lymphoma: the FIL FOLL05 trial. Clinical Cancer Research, 20, 6398-6405.

Gribben, J.G., Freedman, A.S., Neuberg, D., Roy, D.C., Blake, K.W., Woo, S.D., Grossbard, M.L., Rabinowe, S.N., Coral, F., Freeman, G.J., Ritz, J. \& Nadler, L.M. (1991) Immunologic purging of marrow assessed by PCR before autologous bone marrow transplantation for B-cell lymphoma. New England Journal of Medicine, 325, 1525-1533.

Hiddemann, W., Kneba, M., Dreyling, M., Schmitz, N., Lengfelder, E., Schmits, R., Reiser, M., Metzner, B., Harder, H., Hegewisch-Becker, S. Fischer, T., Kropff, M., Reis, H.E., Freund, M., Wormann, B., Fuchs, R., Planker, M., Schimke, J., Eimermacher, H., Trumper, L., Aldaoud, A., Parwaresch, R. \& Unterhalt, M. (2005) Frontline therapy with rituximab added to the combination of cyclophosphamide, doxorubicin, vincristine, and prednisone (CHOP) significantly improves the outcome for patients with advanced-stage follicular lymphoma compared with therapy with $\mathrm{CHOP}$ alone: results of a prospective randomized study of the German Low-Grade Lymphoma Study Group. Blood, 106, 3725-3732.

Hirt, C., Schuler, F., Kiefer, T., Schwenke, C., Haas, A., Niederwieser, D., Neser, S., Assmann, M., Srock, S., Rohrberg, R., Dachselt, K., Leithauser, M., Rabkin, C.S., Herold, M. \& Dolken, G. (2008) Rapid and sustained clearance of circulating lymphoma cells after chemotherapy plus rituximab: clinical significance of quantitative $\mathrm{t}(14 ; 18)$ PCR monitoring in advanced stage follicular lymphoma patients. British Journal of Haematology, 141, 631-640.

Janikova, A., Bortlicek, Z., Campr, V., Kopalova, N., Benesova, K., Belada, D., Prochazka, V., Pytlik, R., Vokurka, S., Pirnos, J., Duras, J., Mocikova, H., Mayer, J. \& Trneny, M. (2015) Radiotherapy with rituximab may be better than radiotherapy alone in first-line treatment of early-stage follicular lymphoma: is it time to change the standard strategy? Leukaemia \& Lymphoma, 56, 2350-2356.

Klein, U. \& Dalla-Favera, R. (2008) Germinal centres: role in B-cell physiology and malignancy. Nature Reviews Immunology, 8, 22-33.

Kluin, P.M. (2013) Origin and migration of follicular lymphoma cells. Haematologica, 98, 13311333.

Ladetto, M., Sametti, S., Donovan, J.W., Ferrero, D., Astolfi, M., Mitterer, M., Ricca, I., Drandi, D., Corradini, P., Coser, P., Pileri, A., Gribben, J.G. \& Tarella, C. (2001) A validated real-time quantitative PCR approach shows a correlation between tumor burden and successful ex vivo purging in follicular lymphoma patients. Experimental Hematology, 29, 183-193.

Ladetto, M., Lobetti-Bodoni, C., Mantoan, B., Ceccarelli, M., Boccomini, C., Genuardi, E., Chiappella, A., Baldini, L., Rossi, G., Pulsoni, A., Di Raimondo, F., Rigacci, L., Pinto, A., Galimberti, 
S., Bari, A., Rota-Scalabrini, D., Ferrari, A., Zaja, F., Gallamini, A., Specchia, G., Musto, P., Rossi, F.G., Gamba, E., Evangelista, A., Vitolo, U. \& Fondazione Italiana, L. (2013) Persistence of minimal residual disease in bone marrow predicts outcome in follicular lymphomas treated with a rituximab-intensive program. Blood, 122, 3759-3766.

Lowry, L., Smith, P., Qian, W., Falk, S., Benstead, K., Illidge, T., Linch, D., Robinson, M., Jack, A. \& Hoskin, P. (2011) Reduced dose radiotherapy for local control in non-Hodgkin lymphoma: a randomised phase III trial. Radiotherapy and Oncology, 100, 86-92.

Luminari, S., Galimberti, S., Versari, A., Biasoli, I., Anastasia, A., Rusconi, C., Ferrari, A., Petrini, M., Manni, M. \& Federico, M. (2016) Positron emission tomography response and minimal residual disease impact on progression-free survival in patients with follicular lymphoma. A subset analysis from the FOLL05 trial of the Fondazione Italiana Linfomi. Haematologica, 101, e66-8.

Mac Manus, M.P. \& Hoppe, R.T. (1996) Is radiotherapy curative for stage I and II low-grade follicular lymphoma? Results of a long-term follow-up study of patients treated at Stanford University. Journal of Clinical Oncology, 14, 1282-1290.

MacManus, M., Fisher, R., Roos, D., O’Brien, P., Macann, A., Davis, S., Tsang, R., Christie, D., McClure, B., Joseph, D., Jayamohan, J. \& Seymour, J.F. (2018) Randomized trial of systemic therapy after involved-field radiotherapy in patients with early-stage follicular lymphoma: TROG 99.03. Journal of Clinical Oncology, 36, 2918-2925.

Mamessier, E., Broussais-Guillaumot, F., Chetaille, B., Bouabdallah, R., Xerri, L., Jaffe, E.S. \& Nadel, B. (2014) Nature and importance of follicular lymphoma precursors. Haematologica, 99, 802-810.

von Neuhoff, N., Dreger, P., Suttorp, M., Marget, M., Kell, S. \& Schmitz, N. (1998) Comparison of different strategies of molecular genetic monitoring following autologous stem cell transplantation in patients with follicular lymphoma. Bone Marrow Transplantation, 22, 161-166.

Pugh, T.J., Ballonoff, A., Newman, F. \& Rabinovitch, R. (2010) Improved survival in patients with early stage low-grade follicular lymphoma treated with radiation: a Surveillance, Epidemiology, and End Results database analysis. Cancer, 116, 3843-3851.

Pulsoni, A., Starza, I.D., Frattarelli, N., Ghia, E., Carlotti, E., Cavalieri, E., Matturro, A., Tempera, S., Rambaldi, A. \& Foa, R. (2007) Stage I/ II follicular lymphoma: spread of bcl-2/IgH+ cells in blood and bone marrow from primary site of disease and possibility of clearance after involved field radiotherapy. British Journal of Haematology, 137, 216-220.

Rambaldi, A., Carlotti, E., Oldani, E., Della Starza, I., Baccarani, M., Cortelazzo, S., Lauria, F., Arcaini, L., Morra, E., Pulsoni, A., Rigacci, L., Rupolo, M., Zaja, F., Zinzani, P.L., Barbui, T. \& Foa, R. (2005) Quantitative PCR of bone marrow $\mathrm{BCL} 2 / \mathrm{IgH}+$ cells at diagnosis predicts treatment response and long-term outcome in follicular non-Hodgkin lymphoma. Blood, 105, 3428-3433.

Reynders, K., Illidge, T., Siva, S., Chang, J.Y. \& De Ruysscher, D. (2015) The abscopal effect of local radiotherapy: using immunotherapy to make a rare event clinically relevant. Cancer Treatment Reviews, 41, 503-510.

Roulland, S., Lebailly, P., Lecluse, Y., Heutte, N., Nadel, B. \& Gauduchon, P. (2006) Long-term clonal persistence and evolution of $\mathrm{t}(14 ; 18)$ bearing B cells in healthy individuals. Leukemia, 20, 158-162.

Ruella, M., Filippi, A.R., Bruna, R., Di Russo, A., Magni, M., Caracciolo, D., Passera, R., Matteucci, P., Di Nicola, M., Corradini, P., Parvis, G., Gini, G., Olivieri, A., Ladetto, M., Ricardi, U., Tarella, C. \& Devizzi, L. (2016) Addition of rituximab to involved-field radiation therapy prolongs progression-free survival in stage I-II follicular lymphoma: results of a multicenter study. International Journal of Radiation Oncology Biology Physics, 94, 783-791.

Sancho, J.M., Garcia, O., Mercadal, S., Pomares, H., Fernandez-Alvarez, R., Gonzalez-Barca, E., Tapia, G., Gonzalez-Garcia, E., Moreno, M., Domingo-Domenech, E., Sorigue, M., Navarro, J.T., Motllo, C., Fernandez-de-Sevilla, A., Feliu, E. \& Ribera, J.M. (2015) The long term followup of early stage follicular lymphoma treated with radiotherapy, chemotherapy or combined modality treatment. Leukemia Research, 39, 853858.
Solal-Céligny, P., Roy, P., Colombat, P., White, J., Armitage, J.O., Arranz-Saez, R., Au, W.Y., Bellei, M., Brice, P., Caballero, D., Coiffier, B., CondeGarcia, E., Doyen, C., Federico, M., Fisher, R.I., Garcia-Conde, J.F., Guglielmi, C., Hagenbeek, A., Haïoun, C., LeBlanc, M., Lister, A.T., LopezGuillermo, A., McLaughlin, P., Milpied, N., Morel, P., Mounier, N., Proctor, S.J., Rohatiner, A., Smith, P., Soubeyran, P., Tilly, H., Vitolo, U., Zinzani, P., Zucca, E. \& Montserrat, E. (2004) Follicular lymphoma international prognostic index. Blood, 104, 1258-1265.

Vargo, J.A., Gill, B.S., Balasubramani, G.K. \& Beriwal, S. (2015) What is the optimal management of early-stage low-grade follicular lymphoma in the modern era? Cancer, 121, 3325-3334.

van der Velden, V.H., Cazzaniga, G., Schrauder, A., Hancock, J., Bader, P., Panzer-Grumayer, E.R., Flohr, T., Sutton, R., Cave, H., Madsen, H.O., Cayuela, J.M., Trka, J., Eckert, C., Foroni, L., Zur Stadt, U., Beldjord, K., Raff, T., van der Schoot, C.E., van Dongen, J.J.; for the European Study Group on MRD detection in ALL (ESG-MRD-ALL). (2007) Analysis of minimal residual disease by Ig/TCR gene rearrangements: guidelines for interpretation of real-time quantitative PCR data. Leukemia, 21, 604-611.

Weinberg, O.K., Ai, W.Z., Mariappan, M.R., Shum, C., Levy, R. \& Arber, D.A. (2007) "Minor" BCL2 breakpoints in follicular lymphoma: frequency and correlation with grade and disease presentation in 236 cases. The Journal of Molecular Diagnostics, 9, 530-537.

Wennekes, L., Ottevanger, P.B., Raemaekers, J.M., Schouten, H.C., de Kok, M.W., Punt, C.J., Grol, R.P., Wollersheim, H.C. \& Hermens, R.P. (2011) Development and measurement of guideline-based indicators for patients with nonHodgkin's lymphoma. Journal of Clinical Oncology, 29, 1436-1444.

Witzens-Harig, M., Hensel, M., Unterhalt, M. \& Herfarth, K. (2011) Treatment of limited stage follicular lymphoma with rituximab immunotherapy and involved field radiotherapy in a prospective multicenter phase II trial-MIR trial. BMC Cancer, 11, 87. 